\title{
Concordant morphologic and gene expression data show that a vaccine halts HER-2/neu preneoplastic lesions
}

\author{
Elena Quaglino, ${ }^{1}$ Simona Rolla, ${ }^{1}$ Manuela lezzi, ${ }^{2}$ Michela Spadaro, ${ }^{1}$ Piero Musiani, ${ }^{2}$ \\ Carla De Giovanni, ${ }^{3}$ Pier Luigi Lollini, ${ }^{3}$ Stefania Lanzardo, ${ }^{1}$ Guido Forni, ${ }^{1,4}$ Remo Sanges, ${ }^{5}$ \\ Stefania Crispi, ${ }^{5}$ Pasquale De Luca, ${ }^{5}$ Raffaele Calogero, ${ }^{1}$ and Federica Cavallo'
}

\begin{abstract}
'Department of Clinical and Biological Sciences, University of Turin, Orbassano, Italy. ${ }^{2}$ Department of Oncology and Neurosciences, G. D'Annunzio University, Chieti, Italy. ${ }^{3}$ Cancer Research Section, Department of Experimental Pathology, University of Bologna, Bologna, Italy. ${ }^{4}$ CERMS, Center for Experimental Research and Medical Studies, Turin, Italy. ${ }^{5}$ Biogem Gene Expression Core Laboratory, Italian Institute for Genetics and Biophysics, Naples, Italy.
\end{abstract}

\begin{abstract}
While much experimental data shows that vaccination efficiently inhibits a subsequent challenge by a transplantable tumor, its ability to inhibit the progress of autochthonous preneoplastic lesions is virtually unknown. In this article, we show that a combined DNA and cell vaccine persistently inhibits such lesions in a murine HER-2/neu mammary carcinogenesis model. At 10 weeks of age, all of the ten mammary gland samples from HER-2/neu-transgenic mice displayed foci of hyperplasia that progressed to invasive tumors. Vaccination with plasmids coding for the transmembrane and extracellular domain of rat $\mathrm{p}^{185^{\text {neu }}}$ followed by a boost with $\mathrm{rp} 185^{\mathrm{neu}}$ allogeneic cells secreting IFN- $\gamma$ kept $48 \%$ of mice tumor free. At 22 weeks, their mammary glands were indistinguishable from those of 10 -week-old untreated mice. Furthermore, the transcription patterns of the two sets of glands coincided. Of the 12,000 genes analyzed, 17 were differentially expressed and related to the antibody response. The use of $B$ cell knockout mice as well as the concordance of morphologic and gene expression data demonstrated that the $\mathrm{Ab}$ response is the main mechanism facilitating tumor growth arrest. This finding suggests that a new way can be found to secure the immunologic control of the progression of HER-2/neu preneoplastic lesions.
\end{abstract}

\section{Introduction}

Ongoing tumor-screening programs are detecting preneoplastic lesions in an increasing number of individuals. Since current treatment is essentially directed to monitoring of disease status and surgical excision, vaccines capable of preventing the progression of such lesions would be an attractive and minimally invasive option (1). However, the use of vaccination in the prevention of the natural progression of an early neoplastic lesion is still a subject rarely addressed, mainly as a result of the considerable difficulty of standardizing appropriate experimental systems.

Transgenic mice that develop autochthonous tumors provide a defined model for assessing the potential of preventive vaccines and the significance of the immune-reaction mechanisms they elicit (2). Moreover, DNA microarray technology can be applied to obtain a genome-wide evaluation of a vaccine's efficacy.

HER-2/neu is an oncogene coding for a $185-\mathrm{kDa}\left(\mathrm{p} 185^{\text {neu }}\right)$ tyrosine kinase receptor involved in cell differentiation, adhesion, and motility. Its low expression in normal tissues and its overexpression

Nonstandard abbreviations used: allogeneic $\left(\mathrm{H}-2^{\mathrm{q}}\right)$ cells expressing rp185 ${ }^{\text {neut }}$ and engineered to release IFN- $\gamma$ (p185 $5^{\text {neu}} /$ alloq-IFN $\gamma$ cells); antibody-dependent cell-mediated cytotoxicity (ADCC); BALB-neuT mice knockout for the Ig $\mu$ chain gene (BALB-neuT/ $\mu \mathrm{KO}$ ); digitized image data (DAT); phycoerythrin (PE); principal component analysis (PCA); product of the rHER-2/neu oncogene (rp185 neu); rat HER-2/neu (rHER-2/neu); rHER-2/neu-transgenic female BALB/c (BALB-neuT); serum binding potential (sbp); spleen cell (Spc); total RNA (ttlRNA); transforming or activated form of rHER-2/nen (rHER-2/nenT); transmembrane and extracellular domain of rp185 $5^{\text {neu }}$ (TM-ECD); two-dimensional (2D); 22-week-old primed-and-boosted BALBneuT mice (wk22pb); 10-week-old untreated BALB-neuT mice (wk10nt); 22-week-old untreated BALB-neuT mice (wk22nt); 2-week-pregnant WT BALB/c mice (wk2prg). Conflict of interest: The authors have declared that no conflict of interest exists. Citation for this article: J. Clin. Invest. 113:709-717 (2004). doi:10.1172/JCI200419850. in $20-30 \%$ of breast cancers, as well as in ovarian, endometrial, gastric, bladder, prostate, and lung cancers, make it an attractive target for active immunotherapy (2). In the rat the WT form of rat HER-2/neu (rHER-2/neu) promotes tumor growth only when it is overexpressed on the cell membrane. By contrast, the transforming or activated form (rHER-2/neuT) displays a mutation at position 664 in the transmembrane (TM) domain (3) that favors the formation of homodimers and heterodimers of the product of the rHER-2/neu oncogene ( $\left.\mathrm{rp} 185^{\text {neu}}\right)$ that transduce proliferative signals responsible for the neoplastic behavior of the cell. Most mice that are transgenic for the rHER-2/neuT derive from the same construct $(3,4)$. Virgin rHER-2/neu-transgenic female BALB/c $\left(\mathrm{H}-2^{\mathrm{d}}\right)$ (BALB-neuT) mice provide one of the most aggressive models of mammary carcinogenesis $(5,6)$, since the product of the rHER-2/ neu oncogene is already overexpressed on the cell surface of the rudimentary mammary glands of 3-week-old females (7). At 6 weeks of age, $\mathrm{rp} 185^{\text {neu+ }}$ cells give rise to side buds that protrude from ductules and form large areas of atypical hyperplasia (8). These progress to multiple in situ carcinomas that enlarge and converge to form a rapidly growing, invasive, and metastasizing tumor palpable between the $22 \mathrm{nd}$ and the 31 st week of age in all ten glands $(5,6,9)$. Repeated vaccination, starting at week 6 , with DNA plasmids coding for distinct portions of $\operatorname{rp} 185^{\text {neu }}(7,10)$ inhibits the onset of preneoplastic lesions. Plasmids coding for the TM and extracellular domain (ECD) of rp185 ${ }^{\text {neu }}$ (TM-ECD plasmids) were the most effective, alone $(7,10)$ or in combination with immunomodulatory molecules $(8,11)$.

To evaluate whether vaccination also hampers the progress of early neoplastic lesions, BALB-neuT mice bearing multiple in situ carcinomas were primed at weeks 10 and 12 with DNA TM-ECD plas- 
mids. Since a subsequent protein boost often enhances the efficacy of DNA vaccination (12-14), groups of these TM-ECD-vaccinated mice were boosted with allogeneic $(\mathrm{H}-2 \mathrm{q})$ cells expressing $\mathrm{rp} 185^{\mathrm{neu}^{+}}$ (15) and engineered to release IFN- $\gamma$ (p185 ${ }^{\text {neu }} /$ alloq-IFN $\gamma$ cells). Previous studies showed that tumor cells engineered to produce IFN- $\gamma$ are especially immunogenic $(15,16)$, while the presence of allogeneic histocompatibility glycoproteins markedly enhances $\mathrm{p} 185^{\text {neu }}$ immune recognition (15). The present paper shows that DNA priming and boosting with allogeneic cells releasing IFN- $\gamma$ halts the progression of mammary carcinogenesis in BALB-neuT mice.

\section{Methods}

Mice. BALB-neuT female mice (H-2d) overexpressing the rHER-2/nen T oncogene under control of the mouse mammary tumor virus promoter (5) were bred under specific pathogen-free conditions by Charles River Italia SpA (Calco, Italy). BALB/c mice knockout for the Ig $\mu$-chain gene, provided by Thomas Blankenstein (Free University of Berlin, Germany) (17), were crossed with BALB-neuT (BALBneuT/ $\mu \mathrm{KO}$ ) and culled by PCR analysis. Only individually tagged virgin female mice were used and treated according to the European Community guidelines. Mammary glands were inspected weekly to note tumor appearance. Neoplastic masses were then measured with calipers in two perpendicular diameters and the average value recorded. Progressively growing masses of greater than $1 \mathrm{~mm}$ mean diameter were regarded as tumors. Differences in tumor incidence were evaluated with the Mantel-Haenszel log-rank test and differences in tumor multiplicity with Student's $t$ test.

Cells. N202.1A and N202.1E cell clones were derived from a mammary carcinoma of FVB-neuN no. 202 mice (H-2q), transgenic for the rHER-2/neu $(15,17)$. Both clones express high levels of H-29 class I but not class II glycoproteins. N202.1A clones highly express membrane $\mathrm{rp} 185^{\text {neu }}$, whereas N202.1E clones are rp185 ${ }^{\text {neu- }}$. N202.1A cells were stably transfected by calcium phosphate precipitation with a plasmid vector carrying the mouse IFN- $\gamma$ gene previously described (16). $\mathrm{p} 185^{\text {neu}} /$ alloq-IFN $\gamma$ cells produced $700 \mathrm{ng} / \mathrm{ml}$ of IFN- $\gamma$ per 24 hours from $1 \times 10^{5}$ seeded cells. The TUBO cell clone was derived from a mammary carcinoma of a BALB-neuT mouse (H-2d) (7). These cells express high levels of both rp185 $5^{\text {neu }}$ and class $I^{d}$, but not class IId, glycoproteins, as previously described in detail (7). Cells were cultured in DMEM (BioWhittaker Inc., Walkersville, Maryland, USA) supplemented with 10\% FBS (Life Technologies Inc., Milan, Italy) at $37^{\circ} \mathrm{C}$ in a humidified $5 \% \mathrm{CO}_{2}$ atmosphere.

Prime and boost vaccination. PcDNA3 vector coding the TM-ECD of rp185 $5^{\text {neu }}$ was produced as described $(7,11)$. It was precipitated, suspended in sterile saline at a concentration of $1 \mathrm{mg} / \mathrm{ml}$, and stored in aliquots at $-20^{\circ} \mathrm{C}$ for use in immunization protocols. A $100-\mu \mathrm{l}$ aliquot of this solution ( $100 \mu \mathrm{g}$ DNA) was injected into the surgically exposed quadriceps of anesthetized mice at weeks 10 and 12 . At 13 weeks of age, mice received an intraperitoneal boost with $2 \times 10^{6}$ p185 ${ }^{\text {neu }} /$ alloq-IFN $\gamma$ cells in $0.2 \mathrm{ml}$ PBS.

Morphologic and immunobistochemical analysis. Groups of three mice were sacrificed at the indicated times, and mammary tissue was processed as previously described $(8,10)$ for histologic, immunohistochemical, and whole-mount analysis (http://ccm.ucdavis.edu/ tgmouse/HistoLab/wholmt1.htm). Plasma cells were counted under a $\times 400$-field microscope $\left(0.180 \mathrm{~mm}^{2}\right)$ in ten randomly chosen fields from each mammary gland sample (ten mammary gland samples per mouse). Morphologic observations were conducted independently by three pathologists in a blind fashion. Differences in plasma cell number were evaluated by the two-tailed Student's $t$ test.
Antibody response. Sera collected from mice at 14 weeks of age were analyzed by flow cytometry as described $(7,15)$. Briefly, $1: 20$ dilutions of sera in PBS-azide-BSA were incubated with $2 \times 10^{5} \mathrm{~N} 202.1 \mathrm{~A} \mathrm{p} 185^{\mathrm{neu}^{+}}$ or N202.1E p185 $5^{\text {neu- }}$ cells for 45 minutes at $4^{\circ} \mathrm{C}$. After washing, the cells were incubated for 30 minutes with rat biotin-conjugated $\mathrm{Ab}$ antimouse total Ig, IgA, IgM, IgG1, IgG2a, IgG2b, IgG3 (Caltag Laboratories Inc., Burlingame, California, USA), and then for 30 minutes with $5 \mu \mathrm{l}$ streptavidin-phycoerythrin (streptavidin-PE) (DAKO A/S, Glostrup, Denmark), resuspended in PBS-azide-BSA containing 1 $\mathrm{mg} / \mathrm{ml}$ of propidium iodide, and evaluated in a FACScan (Becton Dickinson Immunocytometry Systems, Mountain View, California, USA). The specific N202.1A serum binding potential (sbp) was calculated as follows: [(\% positive cells with test serum) (fluorescence mean)] - [(\% positive cells with control serum) (fluorescence mean) $] \times$ serum dilution (22). In each evaluation, $1 \times 10^{4}$ viable cells were analyzed.

Cytotoxicity assay. Spleen cells $\left(\mathrm{Spc} ; 1 \times 10^{7}\right)$ from both TM-ECDvaccinated and primed-and-boosted mice were stimulated for 6 days with $5 \times 10^{5}$ mitomycin-C-treated (Sigma-Aldrich, St. Louis, Missouri, USA) TUBO cells in the presence of $10 \mathrm{U} / \mathrm{ml}$ rat IL-2 (Eurocetus, Milan, Italy) and assayed in a 48 -hour $\left[{ }^{3} \mathrm{H}\right] \mathrm{TdR}$ release assay at effector/target TUBO cell ratios from 50:1 to 6:1 in round-bottomed, 96-well microtiter plates in triplicate. The results were then expressed as $\mathrm{LU}_{20} / 10^{7}$ effector cells (18), with lytic units $20\left(\mathrm{LU}_{20}\right)$ defined as the number of effector cells needed to kill $20 \%$ of the target cells.

IFN- $\gamma$ detection test. Spc were stimulated with Ab's to CD28 and to CD3 $(1 \mu \mathrm{g} / \mathrm{ml}$ final concentration; Pharmingen, San Diego, California, USA) for 3 hours. IFN- $\gamma^{+}$cells were labeled with an $\mathrm{Ab}$ to IFN- $\gamma$ (clone R4-6A2) conjugated with a mAb to CD45 (clone 30S11) (Miltenyi Biotec GmbH, Bergisch Gladbach, Germany) for 5 minutes on ice, then incubated for 45 minutes at $37^{\circ} \mathrm{C}$. Cross-staining was avoided by keeping the density at $1 \times 10^{5}$ cells $/ \mathrm{ml}$. IFN- $\gamma$ bound to the capture matrix was stained with PE-conjugated mAb's to IFN- $\gamma$ (clone AN.18.17.24; Miltenyi Biotec GmbH). Anti-PE microbeads were used to enrich PE-(IFN- $\gamma$ )-stained cells with two rounds on a magnetic separator (MS+ MACS; Miltenyi Biotec GmbH). The cells were counterstained with mAb's to CD4 or CD8 $\alpha$-FITC (Pharmingen) and analyzed by flow cytometry.

Confocal microscopy. TUBO cells were cultured in DMEM at $0.1 \%$ FBS for 24 hours. Cells were washed and incubated for 3 hours at $37^{\circ} \mathrm{C}$ and $4^{\circ} \mathrm{C}$ with sera (diluted $1: 20$ ) derived from immunized mice. Cells were then fixed for 5 minutes with $\mathrm{PBS}-4 \%$ paraformaldehyde (Sigma-Aldrich), permeabilized for 7 minutes with PBS- $0.2 \%$ Triton X-100 (Sigma-Aldrich), and blocked with PBS-10\% BSA (Sigma-Aldrich) for 20 minutes. Membrane and cytoplasmic expression of rp185 ${ }^{\text {neu }}$ was assessed by staining with Alexa Fluor 488-conjugated goat anti-mouse IgG (1 hour, $2 \mu \mathrm{g} / \mathrm{ml}$; Molecular Probes Inc., Eugene, Oregon, USA).

Microarray sample preparation. Total RNA (ttlRNA) was extracted and purified from mammary glands in control and transgenic mice as described in the Affymetrix manual (Affymetrix Inc., Santa Clara, California, USA). ttlRNAs were then spectrophotometrically quantified and inspected by denaturant agarose gel electrophoresis. Lowquality ttlRNAs were discarded, and single-animal ttlRNAs were pooled to obtain three replicates for the mammary glands of 2-weekpregnant WT BALB/c mice (wk2prg), of 22-week-old untreated BALB-neuT mice (wk22nt), and of 22-week-old primed-and-boosted BALB-neuT mice (wk22pb) and two replicates for the mammary glands of 10-week-old untreated BALB-neuT mice (wk10nt). cRNAs were generated and hybridized on 11 MG-U74A v2 Affymetrix DNA chips according to the Affymetrix protocol. ttlRNA $(20 \mu \mathrm{g})$ was used 
for the preparation of double-stranded cDNA using a SuperScript choice system and an oligo(dT) 24 -anchored T7 primer (Invitrogen Corp., Carlsbad, California, USA). The cDNA was then used as a template to synthesize a biotinylated $\mathrm{cRNA}\left(5\right.$ hours, $\left.37^{\circ} \mathrm{C}\right)$ with the aid of the BioArray High Yield RNA Transcript Labeling Kit (Enzo Life Science Inc., Farmingdale, New York, USA). In vitro transcription products were purified on RNeasy spin columns (Qiagen, Hilden, Germany). Biotinylated cRNA was then treated (35 minutes at $94^{\circ} \mathrm{C}$ in a buffer composed of $200 \mathrm{mM}$ Tris acetate $\mathrm{pH} 8.1,500$ $\mathrm{mM}$ potassium acetate, and $150 \mathrm{mM}$ magnesium acetate). Affymetrix 11 MG-U74A v2 array chips were hybridized with biotinylated cRNA $\left(15 \mu \mathrm{g} / \mathrm{chip}, 16\right.$ hours, $\left.45^{\circ} \mathrm{C}\right)$ using the hybridization buffer and control provided by the manufacturer (Affymetrix Inc.). GeneChip Fluidics station 400 (Affymetrix Inc.) was used to wash and stain the arrays. The standard protocol suggested by the manufacturer was used to detect the hybridized biotinylated cRNA. The chips were then scanned with a specific scanner (Affymetrix Inc.) to generate digitized image data (DAT) files.

Microarray data analysis and clustering. DAT files were analyzed by MAS 5.0 to generate background-normalized image data (CEL files). Probe set intensities were obtained by means of the robust multiarray analysis method (19). The full data set was normalized according to the invariant set method (20). The funnel-shaped procedure described by Saviozzi et al. (21) was then applied. The resulting 5,482 probe sets were analyzed by combining two statistical approaches implemented in significance analysis of microarrays (22): two-class unpaired sample method and the multiclass response test (detailed description of the procedure is available at http://www.bioinformatica.unito.it/bioinformatics/Forni/additional_info/ (23). This analysis produced a total of 2,179 probe sets differentially expressed in at least one of the three experimental groups. The validity of the method was demonstrated by real-time RT-PCR evaluation of the expression of several cancer-related genes (see additional information, ref. 23). The 2,179 probe sets were converted in virtual two-dye experiments comparing all replicates of each experimental group with wk 2 prg replicates (i.e., wk $10 \mathrm{nt} j / \mathrm{wk} 2 \operatorname{prg} i$; wk22nt $i / w \mathrm{w} 2$ prgi; wk22pbi/wk2prgi, where $j=1 \rightarrow 2$ and $i=1 \rightarrow 3$ ). Principal components analysis (PCA) (24) and hierarchical clustering were performed on virtual two-dye experiments with a TIGR MultiExperiment Viewer (http://www.tigr.org/software/). Two-dimensional (2D) hierarchical clustering (25) of PCA results was used to identify groups of genes specifically modulated in wk $22 \mathrm{pb}$ only. We used a complete hierarchical clustering together with various distance metrics. The best solution was obtained by Euclidean distance, and genes specifically modulated only in $\mathrm{wk} 22 \mathrm{pb}$ are readily apparent by inspection of the cluster dendrogram (see Figure 5C). Gene ontology classification (26) was performed with the DAVID/EASE annotation tool (http://david.niaid.nih.gov/david/).

Additional information. Additional figures, tables, and normalized microarray data are available at http://www.bioinformatica.unito.it/ bioinformatics/Forni/additional_info/ (23).

\section{Results}

Carcinogenesis inhibition following a prime with TM-ECD plasmids and a boost with $p 185^{\text {neu }} /$ alloq-IFN $\gamma$ cells. Widespread atypical hyperplasia and foci of in situ carcinomas are present in the mammary glands of untreated mice at 10 weeks of age (wk10nt glands; Figure 1A). These carcinomas then grow and converge into fast-growing, invasive, and metastasizing carcinomas that are fully evident by week 22 (wk22nt glands; Figure 1B). All mice display one or more palpable tumors by week 22, and there are palpable tumors in all glands by week 31 (8). Two i.m. TM-ECD vaccinations at the 10 th and 12 th week of age significantly delayed $(P<0.009)$ the occurrence of the first palpable tumor, even though $80 \%$ of vaccinated mice displayed a palpable carcinoma by week 26 (Figure 1D). For more than ten weeks, TM-ECD vaccination also significantly reduced $(P<0.05)$ the number of tumors per mouse (tumor multiplicity) (Figure 1E). This protection was not enhanced by an additional DNA vaccination at week 13 (not shown).

A boost with $\mathrm{p} 185^{\text {neu}} /$ alloq-IFN $\gamma$ cells one week after the TM-ECD prime significantly delayed the development of palpable tumors and kept $48 \%$ of the primed-and-boosted mice tumor-free until week 32 , when the experiment ended (Figure 1D). Tumor multiplicity was also significantly reduced, even when compared to the TM-ECD-vaccinated mice (Figure 1E). The boost was ineffective when administered on its own. All mice displayed palpable tumors by week 22 (Figure 1D).

Immune events induced by prime and boost vaccination. High titers of $\mathrm{Ab}$ to $\mathrm{rp} 185^{\text {neu }}$ were present in the sera of primed-and-boosted mice (Figure $2 \mathrm{~A}$ ). These Ab's were IgG2a and (to a lesser extent) IgG2b, whereas IgG3 was a marginal component (Figure $2 \mathrm{~B}$ ). When tested in vitro, these $A b$ 's efficiently induced internalization of $\mathrm{rp} 185^{\text {neu }}$ from the membrane of rp185 ${ }^{\text {neu+ }}$ tumor cells (Figure 2, D and E). Significantly lower titers were found in the sera of TM-ECD-vaccinated mice. No Ab's were detected in the sera of mice vaccinated with the booster only (Figure 2A).

To assess the significance of $\mathrm{Ab}$ to $\mathrm{p} 185^{\text {neu }}$ in the blockade of carcinogenesis, BALB-neuT/ $\mu \mathrm{KO}$ mice (17) were primed and boosted. The kinetics of the onset of mammary carcinomas was similar in untreated BALB-neuT/ $\mu \mathrm{KO}$ and untreated BALB-neuT mice (not

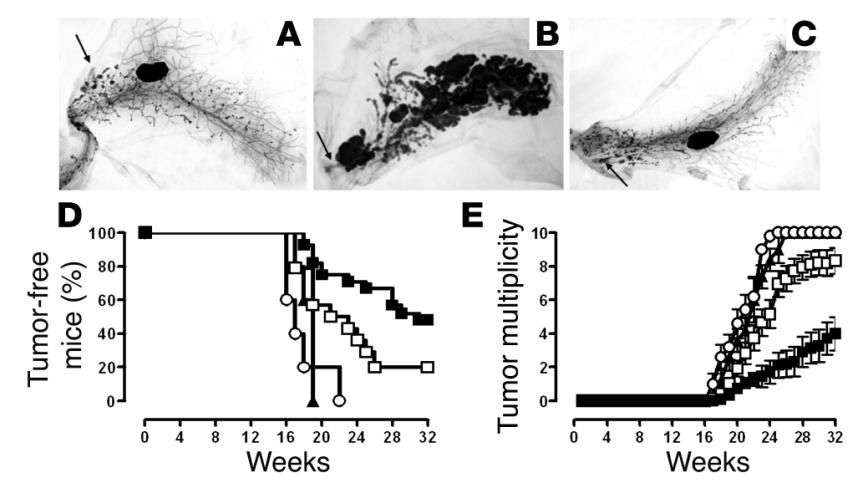

Figure 1

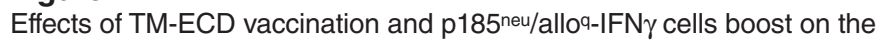
progression of precancerous lesions in BALB-neuT mice. (A-C) Wholemount analysis of mammary glands. The nipple areas are indicated by the arrows. Magnification, $\times 6.3$. (A) Wk10nt mammary glands show several atypical hyperplasia foci forming multiple ductal side buds, sometimes coalescing in larger nodules representing in situ carcinomas. (B) Wk22nt mammary glands; a large portion is occupied by nodular masses corresponding to invasive carcinomas. (C) Wk22pb mammary glands. (D) Percentage of tumor-free mice and (E) tumor multiplicity in BALB-neuT mice. TM-ECD-vaccinated mice (open squares, 18 mice); $185^{\text {neu/alloq-IFN } \gamma}$ cell-vaccinated mice (open circles, 5 mice); primed-and-boosted mice (filled squares, 21 mice); and primed-and-boosted BALB-neuT/ $\mu \mathrm{KO}$ mice (filled triangles, 5 mice). Tumor-free survival curve of primed-and-boosted BALB-neuT mice is significantly different (Mantel-Haenszel test) from that of TM-ECD-vaccinated BALB-neuT mice $(P<0.0001)$ and that of primed-and-boosted BALB-neuT/ $\mu$ KO mice $(P<0.009)$. From week 23 , the mean tumor multiplicity in primed-and-boosted BALB-neuT mice is significantly different from that of TM-ECD-vaccinated mice $(P=0.0002$, Student's $t$ test). Vertical bars represent SE. This experiment was repeated three times. The cumulative data are shown. 

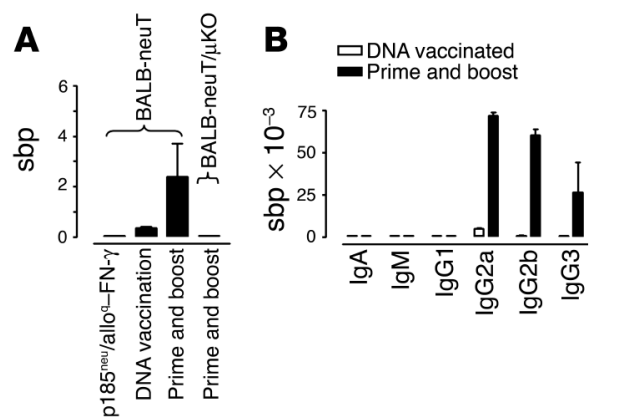

C
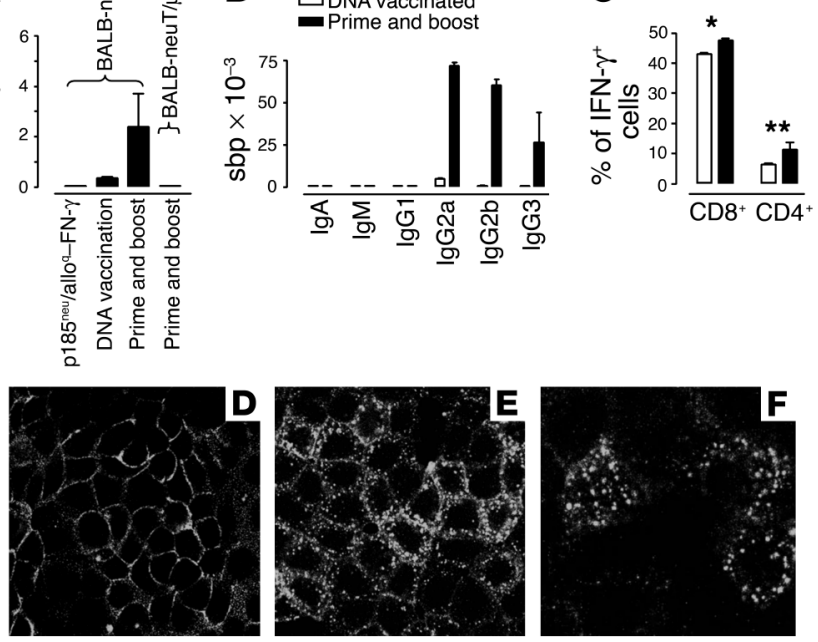

Figure 2

Immune events induced by prime and boost vaccination. (A) Titer of total Ab's to rp185 ${ }^{\text {neu }}$. (B) Titers of Ig isotypes anti-rp185 18 neu in BALB-neuT mice. Values in $\mathbf{A}$ and $\mathbf{B}$ are described as sbp (see Methods section). (C) Percentage of $\mathrm{CD}^{+}{ }^{+}$and $\mathrm{CD} 8^{+}$cells producing IFN- $\gamma$ in the Spc of 22week-old vaccinated mice. Data were obtained in triplicate. ${ }^{*} P=0.0002$; ${ }^{* *} P=0.0073$. (D-F) Ab to rp185 18 neu $d o w n r e g u l a t e s ~ m e m b r a n e ~ r p 185^{\text {neu }}$. rp185 neu+ TUBO cells were incubated with the immune serum from wk22pb mice at $4^{\circ} \mathrm{C}(\mathbf{D})$ and at $37^{\circ} \mathrm{C}(\mathbf{E}$ and $\mathbf{F})$. Large cytoplasmic dots show rp $185^{\text {neu }}$ internalization at $37^{\circ} \mathrm{C}$. These data are combined from three separate experiments. Magnification: $\mathbf{D}$ and E, $\times 100 ; \mathbf{F}, \times 200$.

shown). Even so, priming and boosting did not induce titratable $\mathrm{Ab}$ 's to $\mathrm{p} 185^{\text {neu }}$ (Figure $2 \mathrm{~A}$ ), nor did it afford any protection against carcinogenesis (Figure 1, D and E). Repeated administration of Ab's from primed-and-boosted mice impaired the progression of carcinogenesis in BALB-neuT mice (unpublished observations).

$\mathrm{Spc}$, both freshly isolated and recovered from 6-day cultures with mitomycin-C-inactivated $\mathrm{rp} 185^{\text {neu+ }}$ tumor cells from both TMECD-vaccinated and primed-and-boosted mice, displayed only a marginal cytotoxicity against various $\mathrm{p} 185^{\mathrm{neu}+}$ targets (not shown). Marginal or absent cytotoxic responses were observed when the assays were performed at earlier and later points in time. By contrast, a large number of $\mathrm{CD}^{+}$and a smaller number of $\mathrm{CD}^{+} \mathrm{T}$ cells produced IFN- $\gamma$ after stimulation by mAb's to CD3 and CD28 in Spc from both TM-ECD-vaccinated and primed-and-boosted mice (Figure $2 \mathrm{C}$ ). The percentage of these IFN- $\boldsymbol{\gamma}$-producing T cells was slightly but significantly increased in Spc from primed-and-boosted mice compared with TM-ECD-vaccinated mice.

Morphologic analysis. At week 16, whole-mount assessment disclosed a similar increase of atypical hyperplasia and in situ carcinomas in the glands of TM-ECD-vaccinated mice, primed-and-boosted mice, and untreated controls (not shown). However, from week 18, neoplastic lesions markedly shrank both in TM-ECD-vaccinated mice and in primed-and-boosted mice. A few foci of atypical hyperplasia scattered throughout the gland, and a few nodules corresponding to in situ carcinoma close to the nipple area were evident in wk22pb glands. These lesions were mostly indistinguishable from those of glands from wk10nt mice (Figure 1C). This surprising similarity suggests that vaccination halts carcinogenesis and takes neoplastic lesions back to an early stage. Whereas at week 22 the whole-mount pattern was identi- cal in TM-ECD-vaccinated mice (not shown) and primed-and-boosted mice (Figure 1C), the blockade lasted much longer in the latter group. In addition, plasma cells close to blood vessels $(1.3 \pm 0.6$ cells per microscopic field) and in the delicate stroma surrounding the neoplastic lesions ( $2.3 \pm 0.9$ cells per microscopic field) were present in wk22pb glands (Figure 3B), whereas they were scarce in both TMECD-vaccinated $(0.4 \pm 0.3$ cells per microscopic field) (not shown) and wk $22 \mathrm{nt}$ glands $(0.2 \pm 0.2$ cells per microscopic field) (Figure $2 \mathrm{~A})$. Plasma cells in the mesenchymal tissues of mammary glands of wk22pb mice point to local $\mathrm{Ab}$ production. The presence of $\mathrm{Ab}$ to $\mathrm{p} 185^{\text {neu }}$ in the sera of both TM-ECD-vaccinated and primed-and-boosted mice was associated with downregulation of cell membrane expression of p185 ${ }^{\text {neu }}$ and its confinement in the cytoplasm of neoplastic cells (Figure 3, C and D), as previously observed (7).

Microarray analysis. Four prototypic situations were evaluated: (a) wk10nt glands displaying multifocal preneoplastic lesions (b) wk22nt glands with invasive palpable carcinomas (c) wk22 pb glands that still display preneoplastic mammary lesions, and (d) glands from wk 2 prg mice displaying marked pregnancy-related hyperplasia. The quality of the ttlRNA extracted from lymph node-free mammary tissue from each mouse was assessed on denaturing agarose gel. Biological replicas were generated by pooling the high-quality ttlRNAs from three mice. These pools were used to synthesize biotinylated cRNAs for hybridization on 11 MG-U74A v2 Affymetrix, Inc. GeneChips containing 6,000 sequences present in the mouse UniGene database (build 74) and 6,000 uncharacterized expressed sequence tag clusters. The striking similarity between the gene expression profiles of wk10nt and $w \mathrm{k} 22 \mathrm{pb}$ glands was corroborated by the excellent $r^{2}$ coefficient (Figure 4A), whereas a similar correlation was not obtained when the wk22nt and wk10nt glands were compared (Figure 4B). To identify the genes differentially expressed, a funnel-shaped procedure (21) followed by statistical analysis (22) was applied to the microarray data
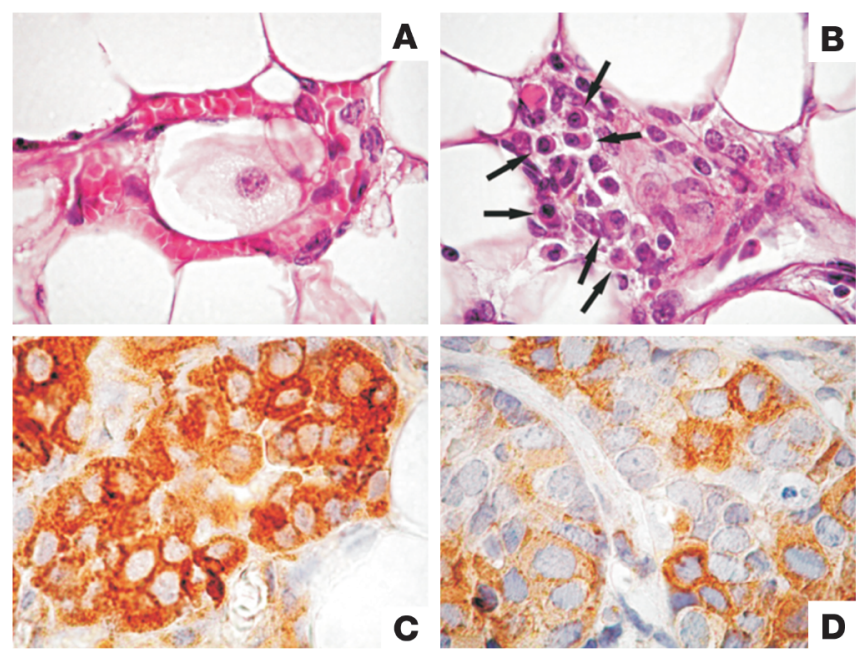

\section{Figure 3}

Plasma cells and p185 18 expression in wk10nt and wk22pb glands. (A) Leukocytes, but not plasma cells, are evident in the tissue from wk10nt glands $(\times 630)$. (B) Numerous plasma cells (arrows) are evident in the delicate stroma close to a small blood vessel of a wk22pb gland $(\times 630)$. (C) p185 18 expression is clearly localized on the membrane and in the cytoplasm of neoplastic cells in wk10nt glands $(\times 1,000)$. (D) $p 185^{\text {neu }}$ expression is markedly reduced and confined to the cell cytoplasm in wk22pb glands $(\times 1,000)$. 
A

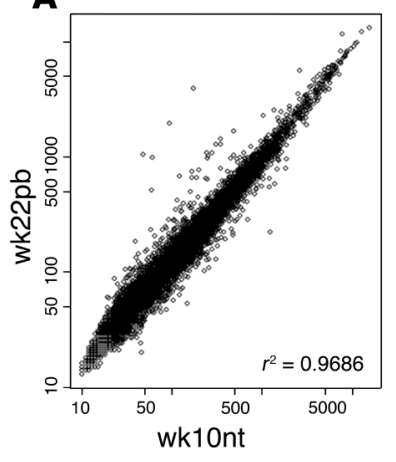

B

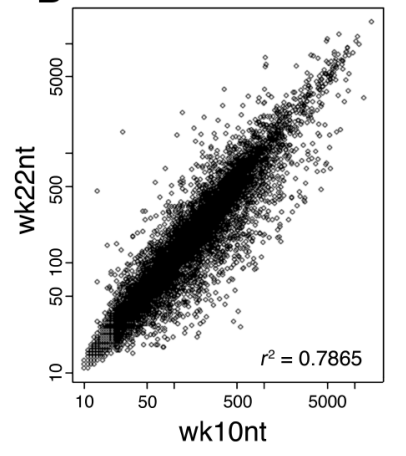

Figure 4

Scattergrams comparing the levels of expression of 12,422 transcripts explored using MG-U74A v2 Affymetrix array in wk10nt, wk22nt, and wk22pb cRNAs from mammary glands. (A) The $r^{2}$ coefficient shows a high correlation between wk22pb and wk10nt data sets. (B) The $r^{2}$ coefficient fails to show any correlation between wk22nt and wk10nt data sets.

set. Statistical validation was obtained for 2,179 differentially expressed probe sets (see additional information, ref. 23).

Hierarchical clustering and PCA. Virtual two-dye experiments (27) were generated by comparing the gene expression linked to HER$2 /$ neu neoplastic alteration of the wk10nt, wk22nt, and wk22 pb glands with that linked to the hormonal hyperplasia of wk2prg glands. The replicates were considered individually to take account of their biological differences. To reduce dimensionality while filtering noise, gene expression patterns were analyzed by PCA (24), an exploratory multivariate statistical technique originally introduced by Pearson (28-30). PCA of gene profiles (Figure 5, A and B) groups the genes in only two large clusters. The $2 \mathrm{D}$ hierarchical clustering analysis (25) shows that in cluster 1 are grouped all probe sets downmodulated in wk22nt with respect to wk22pb (Figure 5C), and in cluster 2 (Figure $5 \mathrm{D}$ ) are grouped all probe sets upmodulated in wk22nt with respect to wk22pb. Furthermore, $2 \mathrm{D}$ hierarchical clustering demonstrates that the expression patterns of wk10nt and wk22pb glands are very similar, especially in PCA cluster 2 (Figure 5D) and clearly distinct from that of wk22nt glands.

Despite this considerable homology of gene expression profiles, however, 69 probe sets, located in five subclusters (Figure 6) were only upregulated in the wk $22 \mathrm{pb}$ glands. All probe sets in clusters a, b, and $\mathrm{d}$ are related to Ig genes (see Table 1). Notably, in cluster $\mathrm{c}$ we have the gene coding for the secretory leukoprotease inhibitor (SLPI; LocusLink ID 20568) whose major physiological role is considered to

\section{Figure 5}

PCA analysis and 2D hierarchical clustering of virtual two-dye experiments. (A) PCA projection showing that the overall analyzed data set is organized in two large clusters on the first two principal components. The two clusters discriminate genes on the expression differences existing between wk22nt mice and the other two groups. In both clusters, wk10nt and wk22pb are grouped together and they are separated from wk22nt. (B) PCA projection showing that the third principal component does not contribute at all to the data clustering. (C) Profiles of PCA cluster $1: 1,122$ probe sets upmodulated in wk22pb/wk10nt with respect to wk22nt; clusters a-e represent the probe sets upmodulated only in wk22pb. (D) Profiles of PCA cluster 2: 1,057 probe sets downmodulated in wk22pb/wk10nt with respect to wk22nt.

be the protection of tissue from proteases at sites of inflammation (31). SLPI is produced by secretory cells in respiratory, genital, and lachrymal glands, and by inflammatory cells that include macrophages, neutrophils, and B cells (31). Related to the presence of inflammatory cells are also proteins coded by genes in cluster e. Of particular importance are EMP3 (LocusLink ID 13732), involved in the inflammatory cascade in monocytes and expressed in adult mouse spleen and thymus (32); the growth factor midkine (LocusLink ID 17242), which promotes migration of macrophages and neutrophils (33); and coronin (LocusLink ID 23789), whose expression is restricted to hematopoietic cells and is involved in the formation of phagocytic vacuoles (34). Also in cluster e is the gene coding for the linker of activation of T cells (LAT; LocusLink ID 16797), one of the most prominent tyrosine-phosphorylated proteins detected following T-cell receptor engagement (35).
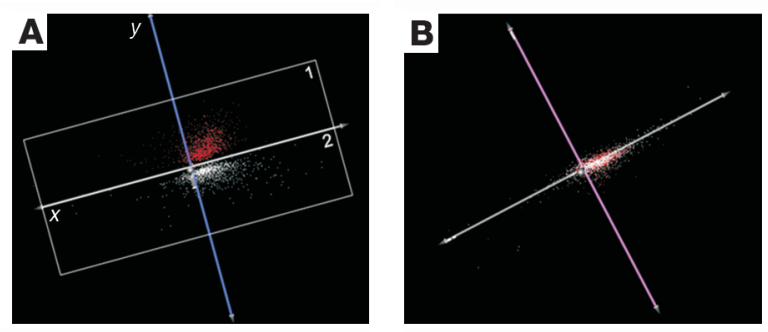

\section{C}

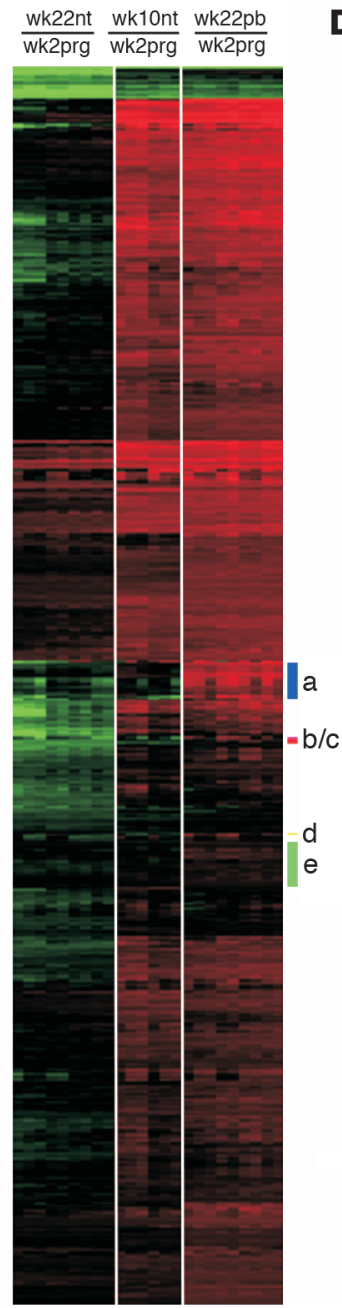

D

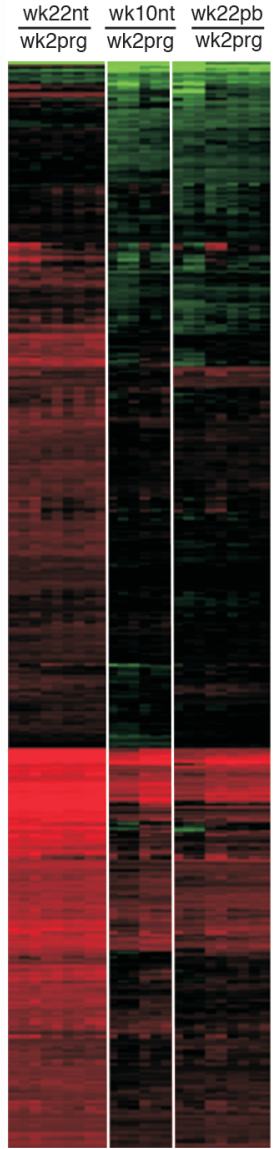


Table 1

List of probe sets found upmodulated in the mammary glands of wk22pb with respect to wk10nt and wk22nt mice

\begin{tabular}{|c|c|c|c|c|}
\hline Cluster & Affymetrix ID & LL ID & Official gene symbol & Description \\
\hline a & 100299_f_at & 16114 & $\lg k-V 28$ & Ig $\kappa$ chain variable 28 (V28) \\
\hline a & 100362 f at & & & Mouse germline IgV $\mathrm{H}_{\mathrm{H}} \mathrm{l}$ gene $\mathrm{H} 8$ \\
\hline a & 100721_f_at & 56304 & & Recombinant antineuraminidase single-chain $\lg V_{H}$ and $V_{L}$ domains \\
\hline a & 101331_f_at & 16123 & $\lg k-V_{8}$ & Ig $\kappa$ chain variable 8 (V8) \\
\hline a & 101633_at & 16097 & $\lg k-\sqrt[V]{20}$ & Ig $\kappa$ chain variable 20 (V20 family) \\
\hline a & 102076_at & & & Mus musculus IgVk aj4 gene \\
\hline a & 102155_f_at & & & Mouse Ig aberrantly rearranged $\kappa$ chain $\mathrm{V} 10-\mathrm{J} 2$ gene ( $\mathrm{V}_{\kappa}-21$ subfamily) \\
\hline a & 102372 at & 16069 & Igj & Ig joining chain \\
\hline a & 102553_at & & & Artificial mRNA for single-chain antibody scFv \\
\hline a & 102823_at & 104961 & AU044919 & Expressed sequence AU044919 \\
\hline a & 102843_s_at & & & Ig heavy-chain 4 (serum IgG1) \\
\hline a & 93904_f_at & & & M. musculus clone N1.1.b lg heavy-chain VDJ region gene \\
\hline a & 93927_f_at & 16017 & $\operatorname{lgh}-4$ & Ig heavy-chain 4 (serum IgG1) \\
\hline a & 96971_f_at & & & Mouse DNA for Ig-א light chain V-J $\kappa 5$ joining region (cell line $\mathrm{CH} 2$ ) \\
\hline a & 96974_at & & & M. musculus IgVк-HNK20 gene \\
\hline a & 97563_f_at & & & M. musculus Ig heavy-chain gene, CDR3 region \\
\hline $\mathrm{a}$ & 97564 f at & & & M. musculus lg $\kappa$ light-chain variable-region gene \\
\hline a & 97574_f_at & 16061 & Igh-VJ558 & Ig heavy chain (J558 family) \\
\hline a & 97711_at & 319900 & B430320C24Rik & RIKEN cDNA B430320C24 gene \\
\hline a & 99369_f_at & & & M. musculus Ig $\kappa$ light-chain variable-region precursor \\
\hline b & 102721_at & 16017 & $\operatorname{lgh}-4$ & Ig heavy-chain 4 (serum IgG1) \\
\hline b & 102722_g_at & 16017 & $\operatorname{lgh}-4$ & Ig heavy-chain 4 (serum IgG1) \\
\hline b & $94725 \mathrm{f}$ at & & & M. musculus Ig $\kappa$ light-chain variable-region precursor (Vk10c) gene \\
\hline c & 92858_at & 20568 & Slpi & Secretory leukocyte protease inhibitor \\
\hline c & 94418 at & 170439 & Elovi6 & ELOVL family member 6 , elongation of long-chain fatty acids (yeast) \\
\hline c & 96144_at & 15904 & Idb4 & Inhibitor of DNA binding 4 \\
\hline d & 98765_f_at & 16061 & $\operatorname{lgh}-$ VJ558 & Ig heavy chain (J558 family) \\
\hline d & 99420_at & 16017 & $\operatorname{lgh}-4$ & Ig heavy-chain 4 (serum IgG1)/M. musculus mRNA (2F7) for IgA V-D-J-heavy chain \\
\hline $\mathrm{e}$ & $101743 \mathrm{f}$ at & 16019 & $\operatorname{lgh}-6$ & Ig heavy-chain 6 (heavy chain of $\mathrm{IgM}$ )/M. musculus mRNA (1B5) for IgA V-D-J-heavy chain \\
\hline e & 102228_at & 16797 & Lat & Linker for activation of $T$ cells \\
\hline e & 102413 at & 109594 & Lmo1 & LIM domain only 1 \\
\hline e & 102924_at & 14357 & Dtx1 & Deltex 1 homolog (Drosophila) \\
\hline e & 103660_at & 18631 & Pex11a & Peroxisomal biogenesis factor $11 \mathrm{a}$ \\
\hline e & 104156_r_at & 11910 & Atf3 & Activating transcription factor 3 \\
\hline e & 104277 at & 56737 & Alg2 & Asparagine-linked glycosylation 2 homolog (yeast, $\alpha$-1,3-mannosyltransferase) \\
\hline e & 104680_at & 51801 & Ramp1 & Receptor (calcitonin) activity-modifying protein 1 \\
\hline e & 104738_at & 22792 & Zrf2 & Zuotin-related factor 2 \\
\hline e & 160098_s_at & 12955 & Cryab & Crystallin, $\alpha$ B \\
\hline e & 160100 at & 27984 & D4Wsu27e & DNA segment, Chr 4, Wayne State University 27, expressed \\
\hline e & 160134_at & 72674 & 2810031L11Rik & RIKEN cDNA $2810031 \mathrm{~L} 11$ gene \\
\hline e & 160244 at & 14154 & Fem1a & Feminization 1 homolog a (Caenorhabditis elegans) \\
\hline e & 160282_at & 66743 & 4931406I20Rik & RIKEN cDNA 4931406120 gene \\
\hline e & 160294 at & 102209 & 0610007H10Rik & RIKEN cDNA $0610007 \mathrm{H} 10$ gene \\
\hline e & 160379_at & 13820 & Epb4.1 & Erythrocyte protein band 4.1 \\
\hline e & 160460_at & 20480 & Skd3 & Suppressor of $\mathrm{K}^{+}$transport defect 3 \\
\hline e & 160561_at & 17242 & Mdk & Midkine \\
\hline e & 160616 at & 24116 & Whsc2h & Wolf-Hirschhorn syndrome candidate 2 homolog (human) \\
\hline e & 160645_at & 11796 & Birc2 & Baculoviral IAP repeat-containing 2 \\
\hline e & 160684_at & 232210 & 8430410A17Rik & RIKEN CDNA $8430410 A 17$ gene \\
\hline e & 162014_i_at & 76281 & 1300011C24Rik & RIKEN CDNA $1300011 \mathrm{C} 24$ gene \\
\hline e & 162177 f_at & 27367 & $R p / 3$ & Ribosomal protein L3 \\
\hline e & 92768_s_at & 11656 & Alas2 & Aminolevulinic acid synthase 2 , erythroid \\
\hline e & 92942_at & 20821 & Trim21 & Tripartite motif protein 21 \\
\hline e & 93047_at & 18141 & Nup50 & Nucleoporin 50 \\
\hline e & 93500_at & 11655 & Alas1 & Aminolevulinic acid synthase 1 \\
\hline e & $93594 r$ at & 13732 & Emp3 & Epithelial membrane protein 3 \\
\hline e & 93666_at & 16909 & Lmo2 & LIM domain only 2 \\
\hline e & 93923_at & 217218 & & Hypothetical protein E030022H21 \\
\hline e & 93966 at & 63958 & Ube4b & Ubiquitination factor E4B, UFD2 homolog (S. cerevisiae) \\
\hline e & 95072 at & 66445 & Crc1 & Cytochrome $c-1$ \\
\hline $\mathrm{e}$ & 95635_g_at & 104457 & 0610010K14Rik & RIKEN cDNA 0610010K14 gene \\
\hline e & 96069_at & 110198 & Akr7a5 & Aldo-keto reductase family 7 , member A5 (aflatoxin aldehyde reductase) \\
\hline e & 96110_at & 12408 & Cbr1 & Carbonyl reductase 1 \\
\hline e & 96219_at & 69780 & 1810031 K02Rik & RIKEN CDNA $1810031 \mathrm{~K} 02$ gene \\
\hline e & 96351_at & 70533 & 4632412E09Rik & RIKEN cDNA 4632412 E09 gene \\
\hline e & 96939_at & 98932 & Myl9 & Myosin, light polypeptide 9 , regulatory \\
\hline e & 98418_at & 13542 & Dvl1 & Dishevelled, dsh homolog 1 (Drosophila) \\
\hline e & 98460 at & 26383 & Fto & Fatso \\
\hline e & 98928_at & 23789 & Coro1b & Coronin, actin-binding protein $1 \mathrm{~B}$ \\
\hline
\end{tabular}




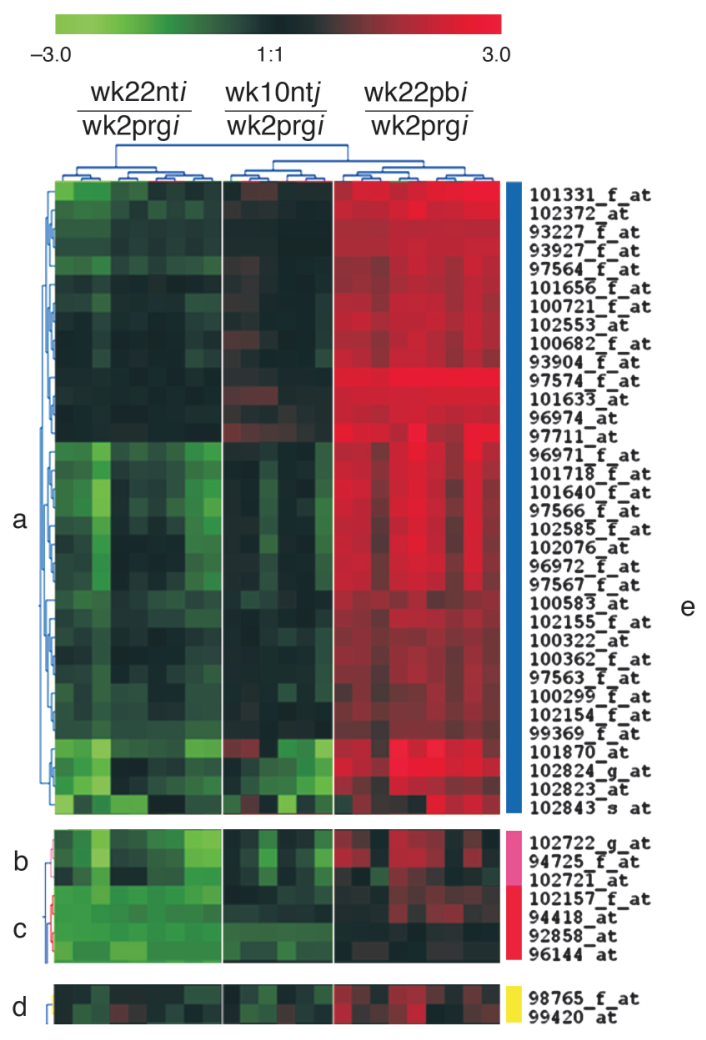

Figure 6

2D hierarchical clustering of PCA cluster 1. A description of the genes queried by the probe sets is given in Table 1.

\section{Discussion}

BALB-neuT female mice are genetically predestined to die because of multiple, fast-growing, invasive, and metastasizing carcinomas that arise in all their mammary glands. At ten weeks of age, atypical hyperplasia and multifocal in situ carcinomas are widespread in all ten glands. The cells of these lesions express $r p 185^{\text {neu }}$ on their membrane, and the proliferation-associated marker in their nucleus (8). At this stage, TM-ECD vaccination does little to delay the appearance of mammary carcinomas, whereas much more effective and prolonged protection is obtained when it is followed by a boost with p185 18 eu $/$ alloq-IFN $\gamma$ cells. About half of the primed-andboosted BALB-neuT mice were tumor-free at week 32 when the in vivo observation was ended. This result suggests that in situ carcinomas are an appropriate target for a specific immune attack and that vaccination against $\mathrm{p} 185^{\text {neu }}$ could become a therapeutic option in women.

Concordant whole-mount morphologic findings and gene expression patterns show that the immune reaction halts the progression of the preneoplastic lesions, and that the mammary lesions revert back to the stage when vaccination began. While the morphologic and gene expression patterns of $\mathrm{wk} 22 \mathrm{pb}$ and wk10nt glands are mostly identical, a group of genes generically pertinent to the humoral response and encoding Ab-related genes is upregulated in the wk22pb glands only. This upregulation correlates well with the presence of plasma cells associated with the wk22 pb glands and suggests production of IgA in the mammary gland. The Ig J polypeptide (LocusLink ID 16069) (Table 1) is one of the genes that is selectively upregulated in $\mathrm{wk} 22 \mathrm{pb}$ glands. The importance of $\mathrm{Ab}$ is further underlined by the

\section{A}

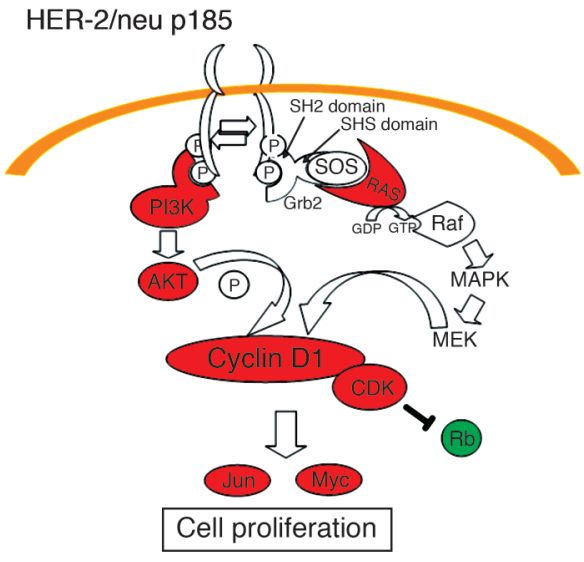

B HER-2/neu p185

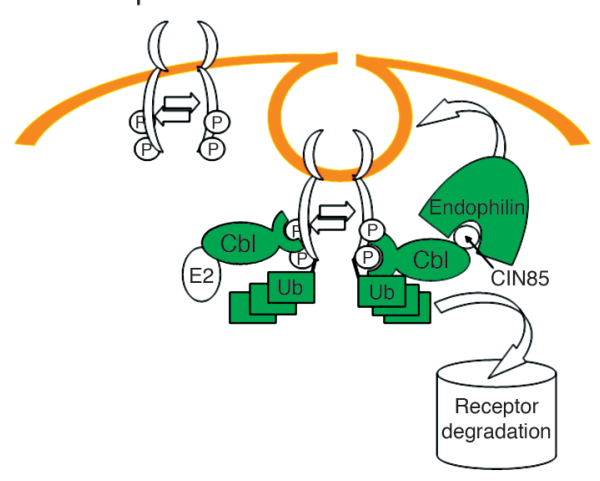

Figure 7

Changes in $185^{\text {neu }}$ signal transduction and degradation pathways in wk22nt as compared to wk10nt and wk22pb glands. (A) In wk22nt glands, genes related to the HER2/neu-mediated cell proliferation encoding for $\mathrm{PI} 3 \mathrm{~K}, \mathrm{AKT}$, and RAS are upmodulated (red), whereas mRNA encoding for $\mathrm{Rb}$ is downregulated (green). (B) p185 ${ }^{\text {neu }}$ degradation pathway. After internalization, $\mathrm{p} 185^{\text {neu }}$ is conveyed to degradation via the ubiquitin (Ub) pathway. Genes essential in degradation ( $\mathrm{Cb}$, endophilin, ubiquitin) are downregulated (green) at the transcriptional level. MEK, MAPK/ERK kinase. 
absence of protection in BALB-neuT/ $\mu \mathrm{KO}$ mice unable to produce $A b$ 's to rp185 $5^{\text {neu }}$ after the prime and boost vaccinations.

The high titer of $A b$ to $\mathrm{rp} 185^{\text {neu }}$ correlates with the downregulation of $\mathrm{rp} 185^{\text {neu }}$ in the cells of mammary lesions and its cytoplasmic confinement $(7,8)$. By hampering the ability of $\mathrm{rp} 185^{\text {neu }}$ to form homodimers and heterodimers that spontaneously transduce proliferative signals (36), and by stripping rp $185^{\text {neu }}$ from the cell membrane causing its internalization in the cytoplasm (37-39) - Ab's inhibit the cell-signaling properties of $\mathrm{rp} 185^{\text {neu }}$. Genes related to the activated state of $\mathrm{p} 185^{\text {neu }}$, such as ras, cyclin D1, cdk4 cyclin-dependent kinase, jun transcription factor, protein kinase C, and PI3K (40), were upregulated in wk22nt compared with wk22pb glands (Figure 6 and additional information, ref. 23), whereas $R b$ was downregulated. By contrast, the transcription of $C b l$, endophilin, and ubiquitin genes, which are related to the HER-2/neu degradation pathway (41), is downregulated in wk22nt in comparison with wk22pb mammary glands.

Ab's to rp185 18 neu also affect tumor growth by mediating antibodydependent cell-mediated cytotoxicity (ADCC) and complement-dependent cytotoxicity (42). Dimeric IgA produced in mammary glands induces phagocytosis, ADCC, respiratory burst, and cytokine release through engagement of the Fc $\alpha$ R1 expressed by leukocytes (43).

In immunized BALB-neuT mice, IgG2a is the dominant isotype of serum Ab's to $\mathrm{rp} 185^{\text {neu }}$. This indicates that vaccination elicits the activation of Thelper cells producing IFN- $\gamma$, the primary switch factor for IgG2a. Following activation by mAb's to CD3 and CD28, many IFN- $\gamma$-producing $T$ cells are present in the spleen of vaccinated mice. These cells contribute to tumor inhibition in several ways other than induction of the Ig isotype switch. Intratumoral release of IFN- $\gamma$ triggers a strong delayed-type hypersensitivity-like reaction with the recruitment of many reactive leukocytes and dendritic cells that is very efficient in tumor debulking and induction of a tumor-specific immune memory $(44,45)$. Moreover, IFN- $\gamma$ released by activated T cells changes the genetic program of tumor cells overexpressing the HER-2/neu oncogene and inhibits their proliferation as well as their production of VEGF, while activating their production of the antiangiogenic monokines IP-10 and MIG (9). PCA shows that IFN- $\gamma$ and IFN- $\boldsymbol{\gamma}$-inducible genes are not upmodulated in the wk22pb glands (see additional information, ref. 23), even if upmodulation of the LAT gene (34) indicates the presence of a discrete amounts of T cells. However, at 22 weeks of age (i.e., about 2 months after the boost), the reactive cell infiltration is reduced and transcription of IFN- $\gamma$ and IFN$\gamma$-inducible genes may have been already switched off and returned to basal levels.

The role of humoral response seems to be particularly prominent because of the double role of $\mathrm{rp} 185^{\text {neu}}$, which is both the target tumor antigen and a membrane-exposed receptor regulating cell growth (2). The importance of Ab's in the clearance of neoplastic lesions may well prove not to be a general model of immunotherapy, given that the inhibition mechanisms involved are not restricted to the direct destruction of the malignant cells. However, a similar vaccination could be considered in the management of early lesions expressing one of the many deregulated oncogenic protein kinase membrane receptors directly involved in cell carcinogenesis (2).

We have previously shown that vaccination is effective against the potential risk of HER-2/neu mammary cancer $(7,8,15)$. Here we show that TM-ECD vaccination followed by a boost with $\mathrm{p} 185^{\text {neu }} /$ alloqIFN $\gamma$ cells permits sustained control of the progression of HER-2/nen precancerous lesions. This heterologous immunization elicits a stronger and more persistent immune response than that achieved by priming and boosting with the same vaccine. As reported for other antigens, DNA priming appears to initiate memory cells, whereas the second immunogen expands the memory response (14). In our experiments, the boost combined three distinct immune stimuli, namely rp185 $5^{\text {neu }}$, allogeneic class I MHC glycoproteins, and IFN- $\gamma$ release. Their separate evaluation showed that all three components are required for the maximum protection. Protection was not or only marginally improved when the two TM-ECD vaccinations were followed by a third, by $\mathrm{H}-2 \mathrm{q}$ allogeneic cells that do not express $\mathrm{rp} 185^{\text {neu }}$ (N202-1E cells), or by syngeneic H-2 ${ }^{\mathrm{d}}$ cells (TUBO cells) engineered to release a similar amount of IFN- $\gamma$ (data not shown).

Allorecognition coupled with $\mathrm{p} 185^{\text {neu }}$ probably induces the death of $185^{\text {neu }} /$ alloq-IFN $\gamma$ cells in a way that facilitates the transfer of cellular antigens (i.e., rp185 ${ }^{\text {neu}}$ ) to host antigen-presenting cells (46), rather than providing the milieu of cytokines produced by alloreactive host lymphocytes (15). Even the immune response elicited by prime and boost does not result in clearance of the neoplastic lesions but simply in the arrest of their progression. Current studies are assessing whether a stronger and more persistent protection can be afforded by repeated prime and boost courses.

These observations provide proof of concept that it is possible to block the progression of early neoplastic lesions by a combined immunotherapy approach, provided that one targets the right antigen. However, it is not clear to what extent these findings can be generalized beyond this particular model, for example, to clinical human ductal carcinomas in situ (DCIS) of the breast, since 34-60\% overexpress $\mathrm{p} 185^{\text {neu }}$ (c-erbB-2) (47). Patients for whom this approach may be desirable might be those who cannot undergo surgery because of concomitant, unrelated conditions. Rather than in newly diagnosed, clinically detectable DCIS, this approach may be more useful to control clinically undetectable DCIS lesions in a postsurgical setting (i.e., as secondary prevention) or as a way to generating an endogenous "Herceptin-like" effect in patients with advanced c-erbB-2+ lesions. This could still be advantageous in comparison with exogenous Ab's, because plasma cells releasing Ab's to p185 ${ }^{\text {neu }}$ may be in close proximity to the target cells. The microarray technology is widely used to improve tumor classification and tailor cancer treatment (48). Ours is the first demonstration of the concordance of morphologic and microarray data in investigation of the mechanisms whereby a vaccine can prevent the progression of precancerous lesions.

\section{Acknowledgments}

We thank Augusto Amici and Stefania Rovero for assistance. This work was supported by grants from the Italian Association for Cancer Research (AIRC), the Ministero dell'Universitá e della Ricerca Scientifica, and the Ministero della Salute, FIRB grants, the University of Torino. It was made possible by the generous gift of $\mathrm{BALB} / \mathrm{c}$ mice, KO for the $\operatorname{Ig} \mu$ chain, by Thomas Blankenstein, Free University of Berlin, Germany.

Received for publication August 20, 2003, and accepted in revised form December 23, 2003.

Address correspondence to: Federica Cavallo, Department of Clinical and Biological Sciences, Ospedale San Luigi Gonzaga, 10043 Orbassano, Italy. Phone: 39-11-670-8119; Fax: 39-11-236-8117; E-mail: federica.cavallo@unito.it.

Raffaele Calogero and Federica Cavallo contributed equally to this work. 
1. Forni, G., Lollini, P.L., Musiani, P., and Colombo, M.P. 2000. Immunoprevention of cancer: is the time ripe? Cancer Res. 60:2571-2575.

2. Lollini, P.-L., and Forni, G. 2003, Cancer immunoprevention: tracking down persistent tumor antigen. Trends Immunol. 24:62-66

3. Muller, W.J., Sinn, E., Pattengale, P.K., Wallace, R., and Leder, P. 1988. Single-step induction of mammary adenocarcinoma in transgenic mice bearing the activated c-neu oncogene. Cell. 54:105-115.

4. Lucchini, F., et al. 1992. Early and multifocal tumors in breast, salivary, harderian and epididymal tissues developed in MMTV-Neu transgenic mice. Cancer Lett. 64:203-209.

5. Boggio, K., et al. 1998. Interleukin 12-mediated prevention of spontaneous mammary adenocarcinomas in two lines of Her-2/neu transgenic mice. J. Exp. Med. 188:589-596.

6. Di Carlo, E., et al. 1999. Analysis of mammary carcinoma onset and progression in HER-2/neu oncogene transgenic mice reveals a lobular origin. Lab. Invest. 79:1261-1269.

7. Rovero, S., et al. 2000. DNA vaccination against rat Her-2/Neu p185 more effectively inhibits carcinogenesis than transplantable carcinomas in transgenic BALB/c mice. J. Immunol. 165:5133-5142.

8. Cappello, P., et al. 2003. LAG-3 enables DNA vaccination to persistently prevent mammary carcinogenesis in HER-2/neu transgenic BALB/c mice. Cancer Res. 63:2518-2525.

9. Cavallo, F., et al. 2001. IL12-activated lymphocytes influence tumor genetic programs. Cancer Res. 61:3518-3523.

10. Di Carlo, E., et al. 2001. Inhibition of mammary carcinogenesis by systemic interleukin 12 or $\mathrm{p} 185$ neu DNA vaccination in Her-2/neu transgenic BALB/c mice. Clin. Cancer Res. 7:830-837.

11. Rovero, S., et al. 2001. Insertion of the DNA for the 163-171 peptide of IL1beta enables a DNA vaccine encoding p185 (neu) to inhibit mammary carcinogenesis in Her-2/neu transgenic BALB/c mice. Gene Ther. 8:447-452.

12. Ramshaw, I.A., and Ramsay, A.J. 2000. The primeboost strategy: exciting prospects for improved vaccination. Immunol. Today. 21:163-165.

13. McShane, H. 2002. Prime-boost immunization strategies for infectious diseases. Curr. Opin. Mol. Ther. 4:23-27.

14. Robinson, H.L. 2003. Prime boost vaccines power up in people. Nat. Med. 9:642-43.

15. Nanni, P., et al. 2001. Combined allogeneic tumor cell vaccination and systemic interleukin 12 prevents mammary carcinogenesis in HER-2/neu transgenic mice. J. Exp. Med. 194:1195-1205.

16. Lollini, P.L., et al. 1993. Inhibition of tumor growth and enhancement of metastasis after transfection of the gamma-interferon gene. Int. J. Cancer. 55:320-329.
17. Qin, Z., et al. 1998. B cells inhibit induction of T celldependent tumor immunity. Nat. Med. 4:627-630.

18. Trinchieri, G. 1989. Biology of natural killer cells. Adv. Immunol. 47:187-376.

19. Irizarry, R.A., et al. 2003. Summaries of Affymetrix GeneChip probe level data. Nucleic Acids Res. 31:e15.

20. Li, C., and Wong, W.H. 2001 Model-based analysis of oligonucleotide arrays: expression index computation and outlier detection. Proc. Natl. Acad. Sci. U. S. A. 98:31-36.

21. Saviozzi, S., Iazzetti, G., Caserta, E., Guffanti, A., and Calogero, R.A. 2003. Microarray data analysis and mining. In Methods in molecular medicine: molecular diagnosis of infectious diseases. J. Decker and U. Reischl, editors. Humana Press Inc. Totowa, Massachusetts. 67-90.

22. Tusher, V.G., Tibshirani, R., and Chu, G. 2001. Significance analysis of microarrays applied to the ionising radiation response. Proc. Natl. Acad. Sci. U. S. A. 98:5116-5121.

23. Quaglino, E., et al. Concordant morphologic and gene expression data show that a vaccine freezes HER-2/neu preneoplastic lesions (additional information page). http://www.bioinformatica.unito.it/ bioinformatics/Forni/additional_info/.

24. Raychaudhuri, S., Stuart, J.M., and Altman, R.B. 2000. Principal components analysis to summarize microarray experiments: application to sporulation time series. In Pacific Symposium on Biocomputing, 2000. World Scientific. Singapore; River Edige, New Jersey, USA. 452-463. SMI Report No. SMI-1999-0804.

25. Eisen, M.B., Spellman, P.T., Brown, P.O., and Botstein, D. 1998. Cluster analysis and display of genome-wide expression patterns. Proc. Natl. Acad. Sci. U. S. A. 95:14863-14868.

26. Ashburner, M., et al. 2001. Gene ontology: tool for the unification of biology. The Gene Ontology Consortium. Nat. Genet. 25:25-29.

27. Kaminski, N., and Friedman, N. 2002. Practical approaches to analysing results of microarray experiments. Am. J. Respir. Cell. Mol. Biol. 27:125-132.

28. Basilevsky, A. 1994. Statistical factor analysis and related methods. John Wiley \& Sons. New York, New York, USA.

29. Everitt, B.S., and Dunn, G. 1992. Applied multivariate data analysis. Oxford University Press. New York, New York, USA.

30. Pearson, K. 1901. On lines and planes of closest fit to systems of points in space. The London, Edinburgh and Dublin Philosophical Magazine and Journal of Science. 2:559-572.

31. Nakamura, A., et al. 2003. Increased susceptibility to LPS-induced endotoxin shock in secretory leukoprotease inhibitor (SLPI)-deficient mice. J. Exp. Med. 197:669-674.

32. Bolin, L.M., et al. 1997. HNMP-1: a novel hematopoietic and neural membrane protein differentially regulated in neural development and injury. J. Neurosci.
17:5493-5502.

33. Stoica, G.E., et al. 2002. Midkine binds to anaplastic lymphoma kinase (ALK) and acts as a growth factor for different cell types. J. Biol. Chem. 277:35990-35998.

34. Okumura, M., Kung, C., Wong, S., Rodgers, M., and Thomas, M.L. 1998. Definition of family of coroninrelated proteins conserved between humans and mice: close genetic linkage between coronin-2 and CD45-associated protein. DNA Cell Biol. 17:779-787.

35. Zhang, W., Sloan-Lancaster, J., Kitchen, J., Trible, R.P., and Samelson, L.E. 1998. LAT: the ZAP-70 tyrosine kinase substrate that links $T$ cell receptor to cellular activation. Cell. 92:83-92.

36. Klapper, L.N., et al. 1997. A subclass of tumorinhibitory monoclonal antibodies to ErbB-2/HER-2 blocks crosstalk with growth factor receptors. Oncogene. 14:2099-2109.

37. Katsumata, M., et al. 1995. Prevention of breast tumor development in vivo by downregulation of the p185(neu) receptor. Nat. Med. 1:644-648.

38. Yip, Y.L., and Ward, R.L. 2002. Anti-ErbB-2 monoclonal antibodies and ErbB-2-directed vaccines. Can cer Immunol. Immunother. 50:569-587.

39. Klapper, L.N., Waterman, H., Sela, M., and Yarden, Y. 2000. Tumor-inhibitory antibodies to HER-2/ErbB2 may act by recruiting c-Cbl and enhancing ubiquitination of HER-2. Cancer Res. 60:3384-3388.

40. Hunter, T. 2000. Signaling -2000 and beyond. Cell. 100:113-127.

41. Oved, S., and Yarden, Y. 2002. Molecular tickets to enter cells. Nature 416:133-136.

42. Sliwkowski, M.X., et al. 1999. Nonclinical studies addressing the mechanism of action of trastuzumab (Herceptin). Semin. Oncol. 26:60-70.

43. Herr, A.B., Ballister, E.R., and Bjorkman, P.J. 2003. Insights into IgA-mediated immune responses from the crystal structures of human FcalphaRI and its complex with IgA1-Fc. Nature. 423:614-620.

44. Musiani, P., et al. 1997. Cytokines, tumor-cell death and immunogenicity: a question of choice. Immunol. Today. 18:32-36.

45. Lollini, P.L., et al. 1995. Systemic effects of cytokines released by gene-transduced tumor cells: marked hyperplasia induced in small bowel by gamma-interferon transfectants through host lymphocytes. Int.J. Cancer. 61:425-430.

46. Sauter, B., et al. 2000. Consequences of cell death: exposure to necrotic tumor cells, but not primary tissue cells or apoptotic cells, induces the maturation of immunostimulatory dendritic cells. J. Exp. Med. 191:423-434.

47. Gullick, W.J. 2002. A new model for ductal carcinoma in situ suggests strategies for treatment. Breast Cancer Res. 4:176-178.

48. Van't Veer, L.J., and De Jong, D. 2002. The microarray way to tailored cancer treatment. Nat. Med. 8:13-14. 